\title{
A Multi-Objective Optimization Approach for Fault-Tolerance Provisioning in Multi-Radio Hybrid Wireless-Optical Broadband Access Networks
}

\author{
G. Schütz \\ Institute of Engineering \\ University of Algarve \\ Campus da Penha, 8005-139 Faro, Portugal \\ gschutz@ualg.pt
}

\author{
N. Correia \\ Center for Electronic, Optoelectronic and \\ Telecommunications (CEOT) \\ University of Algarve \\ Campus de Gambelas, 8005-139 Faro, Portugal \\ ncorreia@ualg.pt
}

\begin{abstract}
Wireless-optical broadband-access networks (WOBANs), currently being deployed at the access section, should integrate fault-tolerance in their design so that geographically continuous wireless coverage can be provided without service breaks. In this paper we propose a joint wireless and optical fault-tolerance planning approach for WOBANs having multiple radios in each router. The problem is formalized, as a multi-objective optimization problem, and a heuristic is proposed to solve this problem. Two fault-tolerance planning scenarios, where gateways are arranged differently in the risk groups, are analysed. It is shown that multi-radio routers can be exploited to improve the performance of WOBANs, providing wireless and optical fault-tolerance. Results also indicate that, when using our approach, a small increase in the number of radios can significantly decrease the total capacity required to provide any degree of fault-tolerance.
\end{abstract}

\section{Categories and Subject Descriptors}

J.m [Computer Applications]: Miscellaneous; D.m [Software] Miscellaneous

\section{Keywords}

wireless-optical, fault-tolerance, access networks, heuristic, multi-objective optimization

\section{INTRODUCTION}

The research on cost-effective infrastructures for access networks is expected to increase in the future and opticalwireless combinations are seen as promising approaches due to the expected bandwidth requirements in the access section. In wireless-optical broadband-access network (WOBAN) architectures the fiber is provided as far as possible from the central office $(\mathrm{CO})$ and then wireless access is provided at the

Permission to make digital or hard copies of all or part of this work for personal or classroom use is granted without fee provided that copies are not made or distributed for profit or commercial advantage and that copies bear this notice and the full citation on the first page. To copy otherwise, to republish, to post on servers or to redistribute to lists, requires prior specific permission and/or a fee.

SAC'10 March 22-26, 2010, Sierre, Switzerland

Copyright 2010 ACM 978-1-60558-638-0/10/03 ...\$10.00. front end, being considered flexible and cost-effective architectures. Because of such excellent compromise WOBANs are being deployed as municipal access solutions [5].

For WOBANs to provide geographically continuous wireless coverage it becomes necessary to provide fault-tolerance. When unlicensed spectrum is used, in wireless networking transmissions (e.g. WiFi), the radio band can be common to other systems and disruption of connections or throughput decrease is possible, affecting all devices within the interference range. At the optical section, fiber cuts will affect the transmission of a set of gateways. WOBANs should be prepared to avoid service breaks and must, therefore, be designed to be fault-tolerant. This paper proposes a heuristic approach for a joint wireless and optical fault-tolerance planning in multi-radio WOBANs. That is, wireless routers are assumed to be equipped with multiple radios that can be tuned to non-overlapping channels.

The paper is organized as follows. Section 2 provides an overview on WOBANs and fault-tolerance related issues. Section 3 presents a multi-objective formalization of the :problem. Section 4 describes the proposed heuristic and Section 5 describes and analyses the results. Section 6 concludes the article.

\section{FAULT-TOLERANCE IN WOBANS}

The architecture of a WOBAN, illustrated in Figure 1, includes a multi-hop wireless mesh network (front end) and an optical access network (back end) that provides connection to the Internet [5]. The dominant technology at the back end is the passive optical network (PON) having optical line terminals (OLTs), located at the $\mathrm{CO}$, and optical network units (ONUs) that provide connection to wireless gateway routers. The wireless infrastructure may use standard WiFi or WiMAX technology for wireless mesh connectivity. Multihop user traffic delivery is supported by the mesh wireless routers and the traffic is sent toward wireless gateway routers connected to the ONUs. An ONU can drive multiple gateways.

In WOBANs two fault-tolerance levels must be considered: $i$ ) wireless fault-tolerance; $i i$ ) optical fault-tolerance. At the optical back end, and according to the fiber cut location, different degrees of fault severity may exist: $i$ ) ONU failure (cut at fiber branch of splitter) means that one or multiple gateways will fail and traffic must be redirected to gateways connected to 'alive' ONUs; ii) OLT failure (cut of 


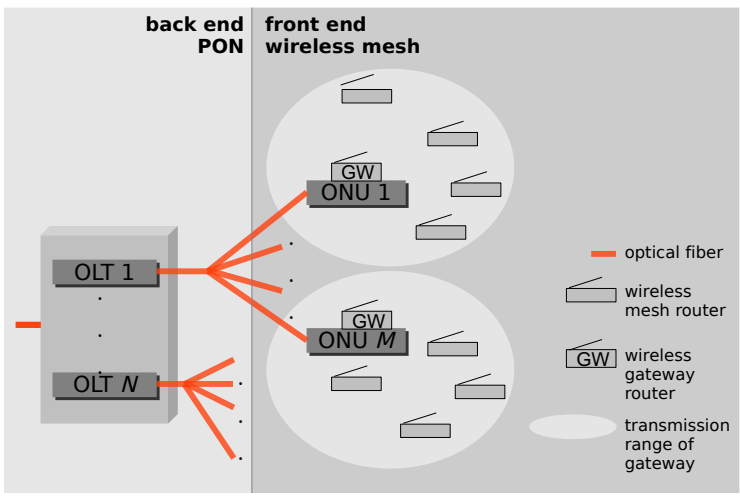

Figure 1: WOBAN architecture.

fiber connected to OLT) means that all ONUs connected to the OLT will fail and traffic must be redirected to gateways connected to 'alive' ONUs and 'alive' OLTs. According to the optical fault-tolerance level being considered, risk groups (RGs) must be defined for gateways. Two gateways belong to the same RG if they share a fiber (main fiber or fiber branch, according to the level of optical fault-tolerance defined) and may, therefore, fail at the same time.

At the front end a wireless link failure is a consequence of unlicensed spectrum utilization, or radio failure, leading to the disruption of connections or throughput speed decrease on devices within the interference range. In high density areas interferences can happen quite frequently and WOBANs must be prepared to work under such environments, providing geographically continuous wireless coverage, so that they can become attractive access solutions.

The survivability approach addopted in this article assumes precomputed primary and backup routes, for every mesh wireless router, using the shortest path. The aim is to properly assign channels to radios in routers, and gateways, used by primary and backup routes so that fault-tolerance is provided (no wireless and/or optical failure can lead to a service break). Primary routes work under normal (no failure) network conditions while backup routes are activated in case of failure. Backup routes are failure dependent, meaning that a backup route is activated for a specific link failure of the primary route it protects. Optical fault-tolerance is provided since primary and backup routes use gateways belonging to different RGs, while wireless fault-tolerance is ensured by proper channel assignment to radios used by primary and backup routes. We assume multi-channel communication at the wireless mesh network, meaning that routers having multiple radio interfaces can simultaneously transmit using non interfering channels (e.g. IEEE 802.11b/g and 802.11a standards provide 3 and 12 orthogonal channels). Two routers may simultaneously transmit using the same channel if they are not at the interference range of each other.

The addopted approach will require a knowledge of the set of nodes (routers/gateways), denoted by $\mathcal{V}$, and set of feasible wireless links $\mathcal{E}$. A node $u \in \mathcal{V}$ can transmit to $v \in \mathcal{V}$, having a feasible wireless link, only if the distance between them is smaller than the transmission range of node $u$. A feasible wireless link becomes a transmission wireless link after channel assignment. The dependence among feasible wireless links is summarized in matrix $I$, as in [2]. When $I\left[l, l^{\prime}\right]=1$ then $l$ can not simultaneously transmit with $l^{\prime}$ (not independent) on the same channel. A matrix $I_{r}$, for router dependence, is also defined where $I_{r}[u, v]=1$ if $u$ interferes with $v$.

The only known previous work on WOBAN fault-tolerance is [4] where an alternative path is provided to 'alive' gateways after failure and no integrated wireless and optical fault-tolerance is performed. Here we provide such integrated fault-tolerance, while assuming multi-radio routers and failure dependent backup routes, not previously assumed in the literature.

\section{MULTI-OBJECTIVE OPTIMIZATION OF SURVIVABILITY PROBLEM IN WOBANS}

\subsection{Multi-Objective Optimization Problems}

A multi-objective optimization problem (MOP) is the problem of determining a vector of decision variables, $X=\left(x_{1}, x_{2}, \ldots, x_{n}\right)$, within a feasible region $S$, while simultaneously minimizing a vector of $k$ objective functions, where $k \geq 2$. Therefore, MOPs can be written in the form:

$$
\operatorname{Min} F(X)=\left[f_{1}(X), f_{2}(X), \ldots, f_{k}(X)\right]^{T},
$$

Subject to $X \in S$.

A solution $X$ is said to dominate another solution $Y$ if and only if $f_{i}(X) \leq f_{i}(Y), \forall i \in\{1, \ldots, k\} \wedge \exists i \in\{1, \ldots, k\}$ : $f_{i}(X)<f_{i}(Y)$. In words, a solution $X$ dominates a solution $Y$ if and only if the solution $X$ is no worse than $Y$ for all objectives and $X$ is strictly better than $Y$ in at least one objective. The notation $X \succ Y$ will be used to indicate that $X$ dominates $Y$.

A solution $X$ is said to be Pareto-optimal if and only if there is no other solution $Y$ that dominates $X$, that is, $X$ is Pareto-optimal if and only if $\nexists Y \in S: Y \succ X$. A Pareto-optimal solution is also called non-dominated solution (NDS).

Usually a MOP has no unique solution but instead a set of non-dominated solutions known as the Pareto-optimal set (or Pareto front). As a matter of fact, if objectives conflict with each other then there are multiple Pareto-optimal solutions, but if objectives do not conflict then there is a unique Pareto-optimal solution [1]. When the conflicting nature of the objectives is not clear, which happens in some problems, a Pareto-optimal set with cardinality one will allow one to conclude that the objectives are not in conflict.

\subsection{Survivability Problem Formulation}

Let us model the network using a directed graph $\mathcal{G}=$ $(\mathcal{V}, \mathcal{E})$, where $\mathcal{V}$ is the set of nodes (routers/gateways) each equipped with one or more interface cards (referred here as radios), and $\mathcal{E}$ is the set of feasible transmission links. For simplicity the transmission and interference ranges are considered to be the same for all radios inside a specific node. The subset of routers aggregating local user traffic, and responsible for injecting the packets into the wireless mesh of the WOBAN, is denoted by $\mathcal{V}_{A} \subset V$. The subset of mesh routers acting as gateways, and attached to an ONU, is denoted by $\mathcal{V}_{G} \subset V$. The set of all risk groups is 
denoted by $\mathcal{R} \mathcal{G}$ while a specific risk group $i$ is denoted by $\mathcal{R G}_{i}$. Risk groups form a partition of $\mathcal{V}_{G}: \bigcup_{i}\left\{u \in \mathcal{R G}_{i}\right\}=$ $\mathcal{V}_{G} \wedge \mathcal{R G}_{i} \cap \mathcal{R \mathcal { G }}_{j}=\emptyset, \forall i, j: i \neq j$. The set of available channels per radio is denoted by $\mathcal{C}$ while the total number of radios in the network is denoted by $R$. A lower bound for $R$ is $R_{L B}=2\left|\mathcal{V}_{A}\right|+\left|\mathcal{V}_{G}\right|$ if backup routes are necessary for all primary routes. The maximum channel transmission rate is normalized as a unit constant and the local traffic demand, at any node $u \in \mathcal{V}_{A}$, is defined proportionally and denoted by $D_{u}$.

Concerning the precomputed primary and backup routes, let us define the following sets:

$\mathcal{P} \quad$ : Primary routes;

$\mathcal{B}^{p}$ : Backup routes protecting a specific primary route $p \in \mathcal{P}$;

$\mathcal{E}^{p} \quad:$ Links used by primary route $p \in \mathcal{P}$;

$\mathcal{E}_{b}^{p} \quad:$ Links used by backup route $b \in \mathcal{B}^{p}$;

$\mathcal{E}^{p, u}:$ Links used by primary route $p \in \mathcal{P}$ having router $u \in \mathcal{V}$ as its source or destination;

$\mathcal{E}_{b, u}^{p}:$ Links used by backup route $b \in \mathcal{B}^{p}$ having router $u \in \mathcal{V}$ as its source or destination.

Every primary route $p \in \mathcal{P}$ or backup route $b \in \mathcal{B}^{p}$, given as input, can be defined as a connected serie of links written as $p: s(p) \in \mathcal{V}_{A} \rightarrow d(p) \in \mathcal{V}_{G}$ and $b: s(b) \in \mathcal{V}_{A} \rightarrow d(b) \in$ $\mathcal{V}_{G}$. Let us define also the following binary variables:

$\tau_{c, l}^{p} \quad: \quad$ If traffic of $p \in \mathcal{P}$ flows through channel $c \in \mathcal{C}$ at link $l \in \mathcal{E}^{p}$, then the variable is equal to 1 ; otherwise, it is equal to 0 ;

$\sigma_{c, l}^{p, b}:$ If backup route $b \in \mathcal{B}^{p}$, protecting primary route $p \in \mathcal{P}$ against the failure of link $l^{\prime} \in \mathcal{E}^{p}: s\left(l^{\prime}\right)=$ $s(b)$, flows through channel $c \in \mathcal{C}$ at link $l \in \mathcal{E}_{b}^{p}$, then the variable is equal to 1 ; otherwise, it is equal to 0 ;

$\rho_{u}^{c} \quad$ : If node $u \in \mathcal{V}$ transmits or receives using channel $c \in \mathcal{C}$, then the variable is equal to 1 ; otherwise, it is equal to 0 .

The decision variables $\tau_{c, l}^{p}, \sigma_{c, l}^{p, b}$ and $\rho_{u}^{c}, \forall p \in \mathcal{P}, \forall b \in$ $\mathcal{B}^{p}, \forall c \in \mathcal{C}, \forall l \in \mathcal{E}^{p} \cup \mathcal{E}_{b}^{p}, \forall u \in \mathcal{V}$ are the components of a solution vector $X$.

When wireless nodes experience channel interference, connection disruption or throughput speed decrease will happen. In fact, all links used by primary routes at the interference range, and using the channel experiencing interference, must activate their backup routes. Under such scenario one objective will be to reduce the impact of a wireless failure. To reach this goal we define function $f_{1}$ that returns the most overloaded link, operating on a specific channel, after the activation of backup routes due to any wireless failure. This objective function is named impact of a wireless failure and is defined as follows:

$$
f_{1}(X)=\max _{c \in \mathcal{C}, l \in \mathcal{E}^{p} \cup \mathcal{E}_{b}^{p}}\left(\alpha_{c, l}+\beta_{c, l}\right)
$$

where

$$
\alpha_{c, l}=\sum_{p \in \mathcal{P}} \sum_{l^{\prime} \in \mathcal{E}^{p}: I\left(l^{\prime}, l\right)=1} \tau_{c, l^{\prime}}^{p} \times D_{s(p)}
$$

accounts for all traffic flowing through primary routes, at the interference range, and

$$
\begin{aligned}
& \beta_{c, l}=\max _{c^{\prime} \in \mathcal{C}, l^{\prime} \in \mathcal{E}}\left(\sum_{p \in \mathcal{P}: \exists l^{\prime \prime} \in \mathcal{E}^{p} \wedge I\left(l^{\prime}, l^{\prime \prime}\right)=1 \wedge \tau_{c^{\prime}, l^{\prime \prime}}^{p}=1}\right. \\
& \left.\sum_{l^{\prime \prime \prime} \in \mathcal{E}_{b}^{p}: s(b)=s\left(l^{\prime \prime}\right) \wedge I\left(l^{\prime \prime \prime}, l\right)=1} \sigma_{c, l^{\prime \prime \prime} \times D_{s(p)}}^{p, b}\right)
\end{aligned}
$$

is the largest amount of traffic that can be forwarded to the interference range (activation of backup routes), at the same time, due to a wireless failure.

Concerning the optical domain, the occurance of an optical failure indicates that all gateways of a risk group are not 'alive' and traffic must be forwarded to gateways belonging to a different risk group. In such failure scenario, the backup routes activated will be the ones protecting the links, of the affected primary routes, ending at any gateway not 'alive'. The other objective, therefore, must be to reduce the impact of an optical failure. To reach this goal, we define function $f_{2}$ that returns the most overloaded link, operating on a specific channel, after the occurence of any optical failure. This objective function is named impact of an optical failure and is defined as follows:

$$
f_{2}(X)=\max _{c \in \mathcal{C}, l \in \mathcal{E}^{p} \cup \mathcal{E}_{b}^{p}}\left(\alpha_{c, l}+\gamma_{c, l}\right)
$$

where $\alpha_{c, l}$ is defined in Eq. 4 and

$$
\begin{array}{r}
\gamma_{c, l}=\max _{\mathcal{R} \mathcal{G}_{i} \in \mathcal{R \mathcal { G }}}\left(\sum_{l^{\prime} \in \mathcal{E}: d\left(l^{\prime}\right) \in \mathcal{R} \mathcal{G}_{i}} \sum_{c^{\prime} \in \mathcal{C}} \sum_{p \in \mathcal{P}: \tau_{c^{\prime}, l^{\prime}}^{p}=1}\right. \\
\left.\sum_{l^{\prime \prime} \in \mathcal{E}_{b}^{p}: I\left(l^{\prime \prime}, l\right)=1} \sigma_{c, l^{\prime \prime}}^{p, b} \times D_{s(p)}\right)
\end{array}
$$

is the largest amount of traffic that can be forwarded to the interference range, at the same time, due to an optical failure affecting one of the risk groups. The variables $\alpha_{c, l}$, $\beta_{c, l}$ and $\gamma_{c, l}$ are float and non negative.

The following MOP formulation allows any fault-tolerance scenario to be implemented:

$$
\operatorname{Min} \quad F(X)=\left[f_{1}(X), f_{2}(X)\right]^{T}
$$

Subject to:

$$
\begin{gathered}
\sum_{c \in \mathcal{C}} \tau_{c, l}^{p}=1, \forall p \in \mathcal{P}, l \in \mathcal{E}^{p} \\
\sum_{c \in \mathcal{C}} \sigma_{c, l}^{p, b}=1, \forall p \in \mathcal{P}, \forall b \in \mathcal{B}^{p}, l \in \mathcal{E}_{b}^{p}
\end{gathered}
$$

$$
\sum_{l^{\prime} \in \mathcal{E}_{b}^{p}: I\left(l^{\prime}, l\right)=1} \sigma_{c, l^{\prime}}^{p, b} \leq\left(1-\tau_{c, l}^{p}\right) \times\left|\mathcal{E}_{b}^{p}\right|, \forall p \in \mathcal{P}, \forall c \in \mathcal{C},
$$$$
, \forall l \in \mathcal{E}, \forall b \in \mathcal{B}^{p}: s(b)=s(l)
$$

$$
\begin{gathered}
\rho_{c}^{u} \geq \frac{\left(\sum_{p \in \mathcal{P}} \sum_{l \in \mathcal{E}^{p, u}} \tau_{c, l}^{p}+\sum_{p \in \mathcal{P}} \sum_{b \in \mathcal{B}^{p}} \sum_{l \in \mathcal{E}_{b, u}^{p}} \sigma_{c, l}^{p, b}\right)}{\left(\sum_{p \in \mathcal{P}}\left|\mathcal{E}^{p, u}\right|+\sum_{p \in \mathcal{P}} \sum_{b \in \mathcal{B}^{p}}\left|\mathcal{E}_{b, u}^{p}\right|\right)}, \forall c \in \mathcal{C}, \forall u \in \mathcal{V}(12) \\
\sum_{u \in \mathcal{V}} \sum_{c \in \mathcal{C}} \rho_{c}^{u} \leq R
\end{gathered}
$$




$$
\begin{array}{r}
\tau_{c, l}^{p}, \sigma_{c, l}^{p, b}, \rho_{c}^{u}, \in\{0,1\}, \forall p \in \mathcal{P}, \forall b \in \mathcal{B}^{p}, \\
, \forall c \in \mathcal{C}, \forall l \in \mathcal{E}, \forall u \in \mathcal{V}
\end{array}
$$

Constraints 9 and 10 allow traffic of primary and backup routes, respectively, to flow through a channel at each link. Constraints 11 do not allow backup and primary routes to use the same channel at links that interfere. Constraints 12 force $\rho_{c}^{u}$ to be one if at least one primary or backup route uses a particular channel, $c$, at some specific node $u$. Constraint 13 limits the total number of radios in the network to $R$. Constraints 14 define binary variables.

\subsection{Problem Properties}

Considering single radio gateway routers, denoted by $g_{i}$, and dual-radio mesh routers, denoted by $u, v$, and any sets of precalculated primary and backup routes, the following properties are enunciated:

Property 1. If gateway routers $g_{i_{1}}$ and $g_{i_{2}}$ interfere, then their receivers must be tuned to different channels. Mesh routers simultaneously adjacent to $g_{i_{1}}$ and $g_{i_{2}}$ must have their radios assigned to those two different channels.

Property 2. If two adjacent mesh routers, $u$ and $v$, are also adjacent to a gateway, $g_{i}$, then the channel assignment of their two radios must be the same.

These two properties allow us to retrieve the following conclusion: if gateway routers $g_{i_{1}}$ and $g_{i_{2}}$ interfere, router $u$ is adjacent to $g_{i_{1}}$ and router $v$ is simultaneously adjacent to $g_{i_{1}}$ and $g_{i_{2}}$, then router $u$ must have the same channel assignment of router $v$. These properties will serve as a basis for the proposed heuristic algorithm.

\section{HEURISTIC ALGORITHM}

There are many approaches to search the Pareto-optimal set. Evolutionary algorithms, for example, can capture the Pareto-optimal set in a single run, since they work with a population of solutions, while heuristics and metaheuristics, like simulated annealing or tabu search, produce only a single solution in each run [1]. Metaheuristics are, however, time-consuming approaches.

Based on the above formulation and properties we implement a local search heuristic to find non dominated solutions (NDS). This heuristic is much faster, when compared with the previously stated approaches. Initially, the heuristic finds a set of solutions for $R_{L B}$ radios considering all different feasible assignments to single radio gateway routers. Then, while incrementing successively the total number of radios, it obtains several solutions performing a local search in the neighbourhood of the most overloaded link of previous non-dominated solutions. To guide local search to different feasible space regions, and to produce dominate solutions, we choose the most overloaded link considering either the wireless or the optical objective. Our heuristic can be described as follows:

\section{Local Search Heuristic}

//It finds the set of non-dominated solutions for $R$ radios, $/ / N D S(R)$, and correspondent $F=\left[f_{1}, f_{2}\right]^{T}$ values.

//First step: solution for $R_{L B}$ radios
$R_{L B}=2\left|\mathcal{V}_{A}\right|+\left|\mathcal{V}_{G}\right| ; N D S\left(R_{L B}\right)=\emptyset$.

Apply Property 1 to find all feasible channel assignments $\left(A_{1}, A_{2}, \ldots, A_{m}\right)$ to the radio of each gateway.

for $i: 1 \rightarrow m$

//Construct a feasible solution $X_{i}$

Make channel assignment to the radio of each gateway $A_{i}$. Apply properties 1 and 2 to assign channels to mesh routers and links reducing the number of free variables.

Sequentially assign feasible channels to still free variables minimizing $\alpha_{c, l}$.

Sequentially assign feasible channels to still free variables minimizing $\beta_{c, l}$ and $\gamma_{c, l}$.

Compute $F\left(X_{i}\right)=\left[f_{1}\left(X_{i}\right), f_{2}\left(X_{i}\right)\right]^{T}$ as defined in (3), (6) and (8).

if $\nexists Y \in N D S\left(R_{L B}\right): Y \succ X_{i}$ then

end for.

$$
N D S\left(R_{L B}\right)=N D S\left(R_{L B}\right) \cup X_{i}
$$

//Second step: solution for increasing number of radios

for $i: R_{L B}+1 \rightarrow R$

$N D S(i)=\emptyset$

for $j: 1 \rightarrow|N D S(i-1)|$

$/ /$ perform a local search

$L S=\emptyset ; X_{j} \in N D S(i-1)$

for $k: 1 \rightarrow 2$

$l=$ most overloaded link for $f_{k}\left(X_{j}\right)$

$L S=\{$ adjacent routers of $u\} \cup\{$ adjacent routers

of $v\}$ where $u, v \in \mathcal{V}: u=s(l) \wedge v=d(l)$

for $q: 1 \rightarrow|L S|$

Add a radio to router $u_{q} \in L S$

Obtain a feasible solution, $Y$, reassigning, in

solution $X_{j}$, channels to primary and backup links adjacent to $u_{q}$ (also reassign router's channels due to eq. 12) minimizing $\alpha_{c, l}$ and: $\beta_{c, l}$ if $k=1$ or $\gamma_{c, l}$ if $k=2$

Compute $F(Y)=\left[f_{1}(Y), f_{2}(Y)\right]^{T}$ as defined

in (3), (6) and (8)

if $\nexists Z \in N D S(i): Z \succ Y$ then

$N D S(i)=N D S(i) \cup Y$

Subtract a radio from router $u_{q} \in L S$

end for.

end for.

end for.

end for.

\section{ANALYSIS OF RESULTS}

\subsection{Network Scenario}

Test data was taken from SFNet, a WOBAN in San Francisco discussed in [3]. This network has 20 wireless mesh routers and 5 wireless gateway routers. The study includes two fault-tolerance scenarios (I and II) having different risk groups for gateways:

Scenario I - Risk groups have cardinality of one: $\mathcal{R} \mathcal{G}_{1}=$ $\{5\}, \mathcal{R G}_{2}=\{13\}, \mathcal{R G}_{3}=\{16\}, \mathcal{R G}_{4}=\{22\}, \mathcal{R G}_{5}=$ $\{25\}$;

Scenario II - Gateways 5 and 13 are connected to the same OLT, gateways 16 and 22 also share an OLT, and gateway 25 shares no OLT: $\mathcal{R G}_{1}=\{5,13\}, \mathcal{R G}_{2}=\{16,22\}$ and $\mathcal{R G}_{3}=\{25\}$.

Precomputed primary routes and link failure dependent backup routes, using the shortest path and having a gateway 
router as destination, were determined and then provided as input to the heuristic proposed. A primary route and its backup routes can not have destination gateway routers at the same risk group, ensuring protection in the case of an optical failure. There are 20 primary routes and 27 backup routes.

Every traffic demand was set to $0.5, D_{u}=0.5, \forall u \in \mathcal{V}_{A}$, assuming that the maximum channel transmission rate is normalized as a unit constant. The available number of independent channels in a router, $|\mathcal{C}|$, was set to 3 , and the maximum value of $R$ was set to 55 . The computational study was carried out on a $3.0 \mathrm{GHz}$ PentiumIV processor with 1 GB RAM. The heuristic code was written in C.

\subsection{Computational Results}

In this section we analyse the results obtained with our heuristic for the multi-objective wireless and optical survivability planning of WOBANs. Heuristic results showing the load of the most overloaded link channel are plotted in Figures 2 and 3 for Scenario I and in Figures 4 and 5 for Scenario II. Figures 2 and 4 show the non-dominated solutions set on both objective functions $\left(f_{1}, f_{2}\right)$ for Scenarios I and II. Figures 3 and 5 plot the load of the most overloaded link channel of non-dominated solutions for each objective separately.

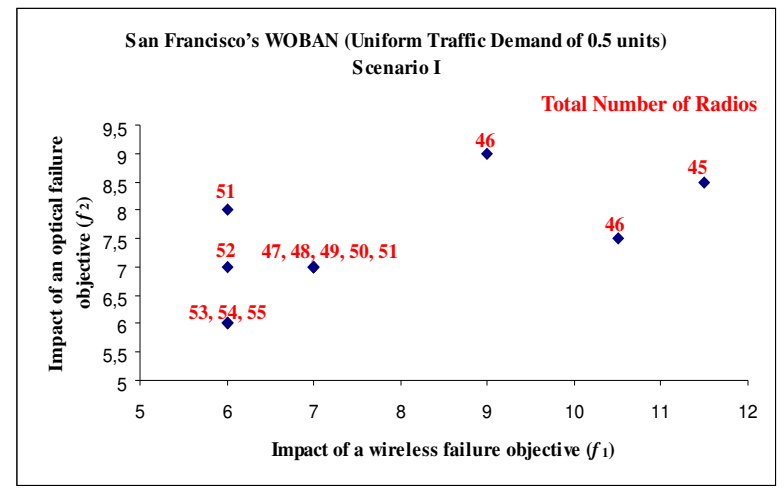

Figure 2: Bi-objective non-dominated solutions for Scenario I.

The values obtained reveal that wireless and optical objectives do not conflict most of the time since, generally, a dominate solution is obtained. In fact, at the end of the local search produced by the algorithm for $R$ available radios, the cardinality of the Pareto-optimal set is usually one. Only in Scenario I, for 46 and 51 radios, this did not occur (see Figures 2 and 4 ).

We emphasize that in Scenario II the wireless and optical failure objectives are plotted almost as parallel lines (Figure 5 ), which reflects the non conflitness of the objectives. Since the optical failure impact is always bigger than the wireless failure impact, the minimization of $f_{2}$ could be enough to find a good solution in this scenario. This behaviour is not present in Scenario I (Figure 3) where the two objective lines are not monotonous and perform some intersections. Initially wireless failures surmount the optical failures but, for 48 or more radios, either the impact of both failures is

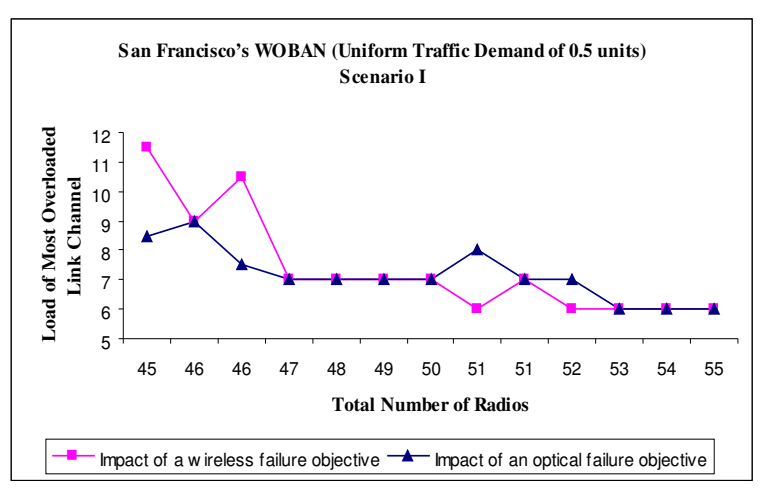

Figure 3: Wireless and optical objectives for Scenario $I$.

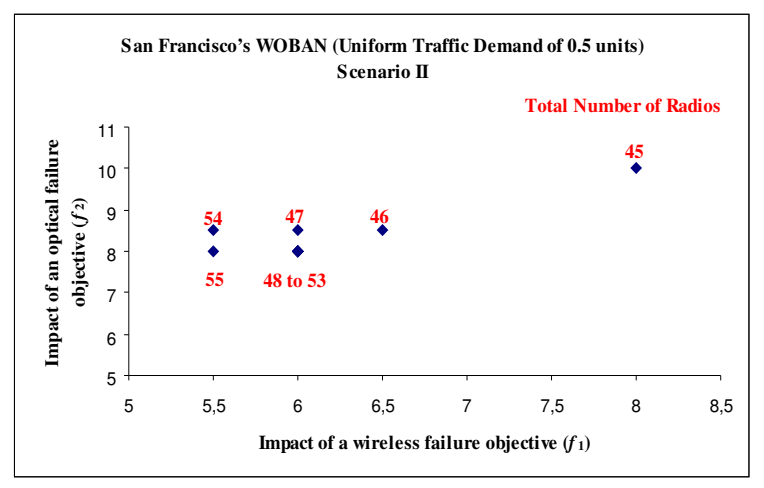

Figure 4: Bi-objective non-dominated solutions for Scenario II.

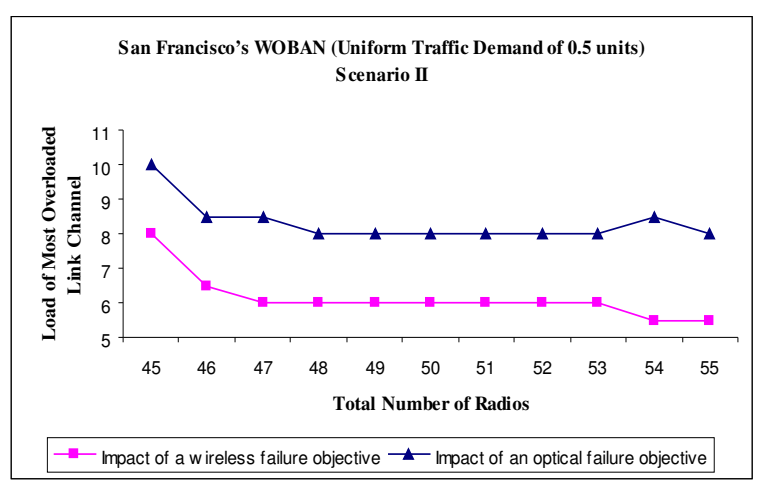

Figure 5: Wireless and optical objectives for Scenario II.

the same or the wireless failure impact is smaller than the optical one.

Our results show that there is a fair load balance among 
links and channels in the network since it was observed that a significant part of the solutions, obtained during the local search, have equal objective function values although related to different links/channels. This may be due to the fact that a load improvement in a link/channel leads to the forwarding of its extra load to other links/channels, meaning that one of those becomes then the most congested. This happens also from 47 to 50 radios and from 53 to 55 radios, for Scenario I (Figures 2 and 3), and from 48 to 53 radios, for Scenario II (Figures 4 and 5), where there is no improvement on both objectives.

The values in all Figures indicate that a small increase in the number of radios (two or three radios) can significantly decrease the total capacity required to provide wireless and optical fault-tolerance, in both scenarios.

In what concerns to wireless failures, Scenario I requires more capacity than Scenario II for 45 to 47 radios but it is almost equal from 51 radios on. Regarding optical failures, on the opposite way, Scenario II requires more capacity than Scenario I, for any number of available radios. This may be explained by the fact that when an optical failure occurs, in Scenario II, traffic flowing through all links of primary routes ending in any of the gateways of a risk group is forwarded to gateways belonging to other risk group, while in Scenario I only one gateway has its traffic redirected.

The heuristic approach took only 0.094 and 0.093 seconds of computational execution time for Scenario I and II, respectively. So, it can be used to solve problems in real-time.

\section{CONCLUSIONS}

This paper shows that the use of multi-radio routers can be exploited to improve the performance of WOBANs providing wireless and optical fault-tolerance. The problem of planning a wireless and optical fault-tolerant multi-radio WOBAN is formulated, as a constrained multi-objective optimization problem, and a local search heuristic developed to capture non dominated solutions. The analysis of results indicate that, when using our approach, a small increase in the number of radios can significantly decrease the total capacity required to provide any degree of fault-tolerance. This is achieved with an efficient spectrum reutilization. Our two objectives aim to reduce, on one hand, the impact of a wireless failure and, on the other, the impact of an optical failure. The heuristic results revealed that these objectives are not conflicting. As a consequence, it is possible to obtain a solution (a set of routes and channel assignments) that provides an efficient answer to any failure impact, being it a wireless or optical failure.

\section{ACKNOWLEDGMENTS}

This work was supported by the Foundation for Science and Technology from Portugal within CEOT (Center for Electronic, Optoelectronic and Telecommunications) and by the project PTDC/EEA-TEL/71678/2006.

\section{REFERENCES}

[1] K. Deb. Multi-Objective Optimization using Evolutionary Algorithms. John Wiley \& Sons, 2001.

[2] J. Zhang, et al. Joint Routing and Scheduling in Multi-Radio Multi-Channel Multi-hop Wireless Networks. International Conference on Broadband Networks, 1:631-640, October 2005.
[3] S. Sarkar, et al. DARA: Delay-Aware Routing Algorithm in a hybrid wireless-optical broadband access network (WOBAN). IEEE ICC, pages 2480-2484, June 2007.

[4] S. Sarkar, et al. RADAR: Risk-and-Delay Aware Routing Algorithm in a hybrid wireless-optical broadband access network (WOBAN). IEEE/OSA OFC, March 2007.

[5] S. Sarkar, et al. A Novel Delay-Aware Routing Algorithm (DARA) for a Hybrid Wireless-Optical Broadband Access Network (WOBAN). IEEE Network, 22(3):20-28, May/June 2008. 